\title{
Using Navies Bayes Algorithm InSentimental Analysis To Improve Business Intelligence
}

\author{
ANKIT TIWARI M.Tech Scholar, Computer Science \& Engineering \\ Dr. BRAJESH KUMAR SINGH Professor \& Head, Department of \\ Computer Science \& Engineering, R.B.S Engineering Technical \\ Campus, Bichpuri, Agra, U.P., India
}

\begin{abstract}
A study of sentimental analysis is opinion mining, feeling, emotions, sentiments mining and sentiments extraction has increased its acceptance some year ago. NowaDays Online survey reviews have become very essential criteria for checking the status of a company. Now In, This research paper we represent a sentimental analysis method to company reviews organization through extensive reviews datasets which are given by Yelp, Yelp Challenging datasets. In the search paper, we represent many techniques for automatic sentimental analysis classification, by using two methods for extraction methods and by using four techniques in machine learning models. It reflects the similar research on the influence of the ensemble techniques for reviewing the sentimental analysisclassification.
\end{abstract}

Keywords Unsupervised learning, Twitter, Maximum Entropy, Support Vector Machine, Sentiment analysis, Naive Bayes, Supervised learning, Opinion Mining

\section{INTRODUCTION}

Now, the time of the Internet has modified the process to represent their people's opinions and people's conclusions. This means almost done by blogger sites, e- commerce sites, social media platforms, and many more. In today's world, most peoples are used by social networking sites like Fb, Instagram, and Twitter, etc. for expressing their sentiments, feeling, opinions, and give their daily life information. With the help of online medium of many, communities we keeps touching with communication sites anywhere user's information and inspire another people by discussions. A larger volume of sentiment-rich data is generated by social media sites like peoples tweets, through status, posting in blogs, commenting and giving reviews, and so on. Though, social communication sites provide a chance for your businesses by providing a program to attach with their right buyers for promotion. Most personalities depend upon client-generated data through online to a wide range for taking decision. E.g. if somebodylike to buysome material or wants to use any service, then they primarilychecktheir surveys online, get information through online after that take decision. The quantity of information which is given by users is enoughto analyzing of another user. So it's very important to automatically test this, are widely there are vast varieties of sentiment analysis technique which are used. It tells users either the given information related the product is correct or not before they purchase it. Companies and Business online also use it for analyzing their material or services in that manner they give opportunities according to user's need. Textual Data retrieval methods mostly trying investigating, exploring, and processing the actual information present. Facts mostly contain actual components but, they also contain some other textual contents that denote subjective features. Those mostly containing opinion mining, sentiment analysis, feelings, and reactions, which determine the core segments of Sentimental Analysis. It provides various difficulties possibilities for the growth of new features, mainly developed because of large promotion of available data insocial media like posting in blogs and many more social networking sites.Example, the guidance of products is represented by the reference of the operation 
which can be predicted whether the opinion about those items is positive or negative by executing the use of Sentiment analysis.

SENTIMENTAL ANALYSIS It is a process that detects automatically feelings, data mining of characters, feelings, viewpoints \& sentiments from words, conversation, tweets, and dataset references by Natural Language Processing. The sentimental analysis contains a distribution of conclusions in a topic into levels such as "positive", "negative" and "neutral". It's applied to subjective review, sentiment mining, and appraisal extraction. The lines or expressions of opinion mining, sentiments analysis, views, and acceptance are used interchangeable but there are variances among them.

- Conclusion: A outcome words (As various experts invention have many opinions expression)

- Representation: Personalview

- Acceptance: Contemplate approval and intellectualpermission

- Sentiments: view expressing one'sopinion

- Result: "Positive" or"Negative"

An example for using terminologies for based on Sentiment Analysis is as given below, $\langle$ SENTENCE $>=$ Iphone 11 mobile phone is very good and I am happy all features very good $\langle$ EMOTION MAKER $>=\langle$ user $>$

$\langle$ OBJECT $\rangle=\langle$ iphone11 $>$

$<$ FEATURE $>=\langle$ mobilephone $>$

$\langle$ FEELINGS $>=\langle$ verygood $>\langle$ happy $>$

$\langle$ POLARITY $>=<$ negative $>$

Sentiment Analysis is a way that combines different types of responsibilities such as sentiment extraction, sentiment classification, subjectivity classification, summarization of views, or conclusion spam detection, between others. It is the main aim to analyze people's thoughts, attitudes, perspectives, feelings emotions, etc.

\section{RELATED WORKS}

Early, most of the research papers related to product reviews, emotion analysis, or opinion mining have been performed recently. In the work [1] Elli, Maria, and Yi-Fan extracted sentiment from the reviews and investigate the outcomes to arise up a business model. They have required that demonstrated mechanisms were strong and adequate to give them high accuracy results is helpful. The method of business analytics helps make decisions more appropriately. They also contributed to detecting emotions from reviews of persons on the names, also detecting fake reviews. They mainly applied two mechanisms MNB means that Multinomial Naive Bayes and SVM meansthatsupportvectormachineastheirmaintaskofclassifiers.Inthisresearchpaper

[2] the author implemented existing supervised learning algorithms to predict a review rating on a given differential measure using only text. They have applied hold-out cross- validation utilizing $75 \%$ data as training data and 25\% data as testing data. In this research paper [3] the author implemented and enhancing the present work in the area of natural language processing and sentiment analysis to data from E-commerce review datasets. Naive Bayes algorithm and decision list classifiers were used to marked the provided review whether positive or negative. In this research paper [4] the author purpose to develop a featureto show the opinion of the reviews in the form of 
graphs. They have related data scraping from the e-commerce site's URL to get the data and preprocessed it. In this research paper, they used the Maximum Entropy, Support Vector Machine, and Naive Bayes Algorithm. They represent their outcomes in the statistical graph. These system tested used bigrams and unigrams. They utilized a subset of reviews sites, games, videos, movies, peoplereviews from UCSD Time-based models didn't work properly as the variation in common ratings amongevery day, month or year was comparetively short. It represents the effects Between bi-grams and uni-grams provided the most correct result. It very important and famous unigrams were anmostly useful predictor for ratings for their large variance. Unigram results performance is better than bigrams.

In our system, we used a wide quantity of datasets so it performed effective results and we could present more reliable decisions. mostly, we used an active learning methods to mark datasets that can dramatically runvarious machine learning work. Our system also includes of various classes of feature enhancingapproach. According to our knowledge, this proposed method gave higher correctness than the present researchworks.

\section{EVALUATION AND RESULTS}

During the evaluation from the individual work of the recommended feature enhancing algorithms and various classifiers be use the correctness which shows the percentage of test units that are classified perfectly from all other test units. We created a structure of the system to explore various strategies in feature enhancingapproach in combination with several classifiers which are explain in section III. In the 1st analysis, we use a unit of 10000 business reviews dataset which is taken from the refined data with the help of Yelp Challenge Dataset. Even we also break these datasets into 80\% for practicing and $20 \%$ for checking. As shown in Table I, We saw in fig that the best accuracy score on the test dataset was performed by the system using $92.6 \%$ of the first method which is given in section III - B, when the preprocessing level is taken before the feature selection (removing punctuations) and handling negations, apply to stem, remove stop words and use unigrams for creating the feature vectors in the bag of texts presentation. Then the result is obtained by using the Stochastic Gradient Descent Classifier with the following values, the squared Euclidean norm L2 which is used for a penalty is merged to the lost function, and a value of 0.0001 of the constant for multiplying in terms of regularization.

TABLE No.1: FEATURES CLASSIFIER ACCURACY

\begin{tabular}{|l|l|l|l|l|}
\hline S.No. & Name Of Algorithm & $\begin{array}{l}\text { Accuracy } \\
\text { Stage 1 }\end{array}$ & $\begin{array}{l}\text { Accuracy } \\
\text { Stage 2 }\end{array}$ & Accuracy Stage \\
\hline 1. & Naïve Bayes & $87.41 \%$ & $88.98 \%$ & $90.14 \%$ \\
\hline 2. & Linear Support Vector & $89.39 \%$ & $90.46 \%$ & $91.37 \%$ \\
\hline 3. & Logistic Regression & $86.92 \%$ & $88.32 \%$ & $86.94 \%$ \\
\hline 4. & Stochastic Gradient Descent & $88.28 \%$ & $88.98 \%$ & $91.87 \%$ \\
\hline
\end{tabular}




\section{Denoted:- (NB) Naïve Bayes, (LSV) Linear Support Vector, (LR) Logistic Regression, (SGD)}

\section{Stochastic Gradient Descent}

By using this very feature set in the algorithm also implementing Linear Support Vector Classifier we received a concluded score (89.39\%), but if we perform the Naive Bayesian for the classification of negative and positive sentimental then this system worked some more critical (90\%). We received a related correctness score $(90 \%)$ when we use a Logistic Regression Classifier we received a concluded score $(86.92 \%)$, for the closing of criteria. In the case of without using any level of handling negations in the feature choosing algorithm the systems of correctness, despite the classifier, we use reductions by $1.0 \%$.

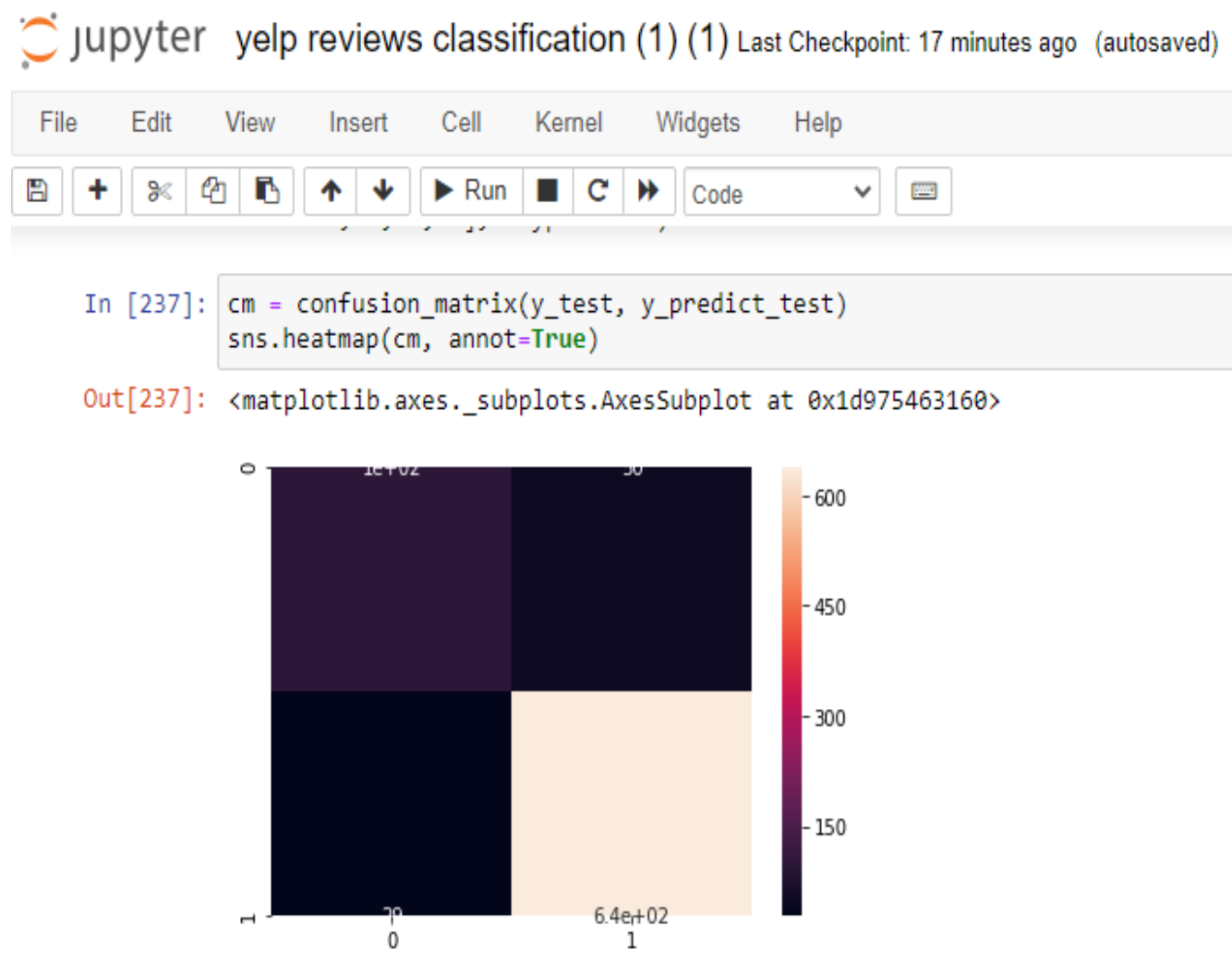

Figure 1: Predict Test Graphically Presentation

So we said that negations handling is a very significant level in sentiment analysis is verified. We previously verified that we don't delete punctuations from the document review in the functioning stage, but by using the handling negations, the correctness lightly reduces besides. 


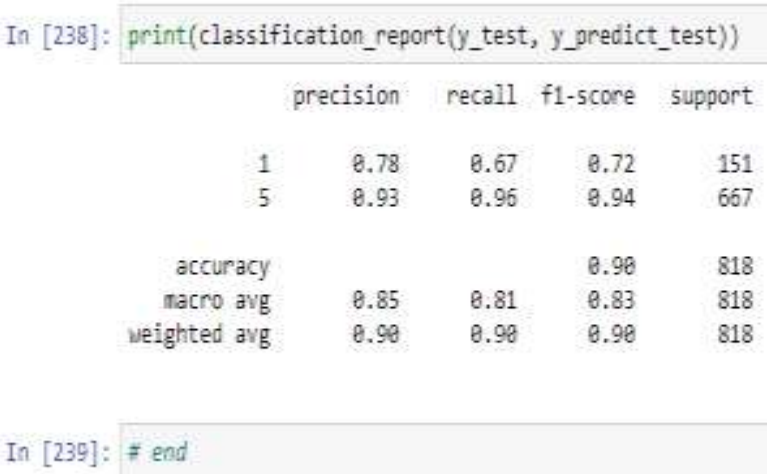

Figure 2: Data classifying and f1 check - the harmonic mean of precision and recall

We estimate (Table No.2) the reflection of the learning algorithms utilizing the 1st method of the feature enhacing algorithm based on precision, f1-score, support and recall, the 1st for positive data classifying, and the other for negative data classifying and $\mathrm{f} 1$ check - the harmonic mean of precision and recall (1.0 is the best value).

TABLE No 2: CLASSIFIER OF F-MEASURE, PRECISION AND RECALL

\begin{tabular}{|l|l|l|l|l|}
\hline Features & Precision & recall & F1-score & support \\
\hline Naïve Bayes & 0.90 & 0.90 & 0.90 & 818 \\
\hline $\begin{array}{l}\text { Linear Support } \\
\text { Vector }\end{array}$ & 0.85 & 0.81 & 0.83 & 818 \\
\hline $\begin{array}{l}\text { Logistic } \\
\text { Regression }\end{array}$ & 0.78 & 0.67 & 0.72 & 151 \\
\hline $\begin{array}{l}\text { Stochastic } \\
\text { Gradient } \\
\text { Descent }\end{array}$ & 0.93 & 0.96 & 0.94 & 667 \\
$\begin{array}{l}\text { Denoted:- (NB) Naïve Bayes, (LSV) Linear Support Vector, (LR) Logistic } \\
\text { Regression, (SGD) } \\
\text { Stochastic Gradient Descent }\end{array}$ & &
\end{tabular}

\section{Figure 2.0.}

The results we obtained on the test dataset are as follows. We show that the 2nd method of characteristic extraction, by applying (P.O.S) means that the part of speech, by using (W.S.D) means that word sense disambiguation, and by estimating is either positive or negative score applying the sentiment opinion lexicon-based algorithm were not much beneficial. The correctness of the system for every classifier was reduced by $14 \%$ if we compared it to the 1 st method outcomes. We too show that the working is reduced during cross-validation, and therefore we say that certain features were not involved in the next analyses. Although this method need not any practining compared with the 1st method, we know that the sense of the full-data review may be huge and it is too widely depend on the content. Sentiment analysis can happen, example, a statement of a data review that contains a positive or negative report doesn't need to show any 
sentiment (for example- "you recommend a good restaurant close to this company"). Succeeding, for the evaluation of the correctness of the recommended techniques, we used the test business reviews dataset.

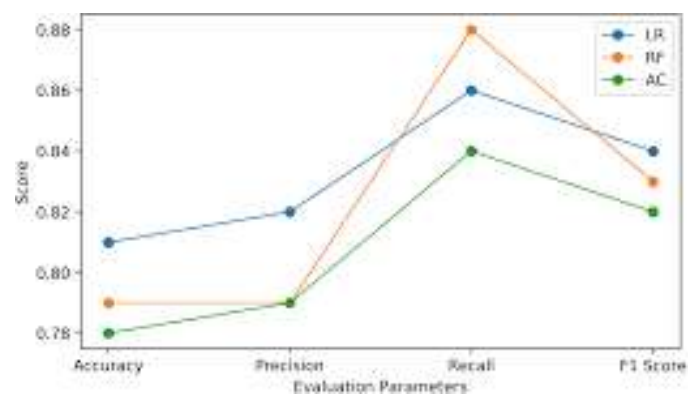

Figure 3.0. Grading of Correctness of Parameters opinion

A comparison of correctness on the test database applying various learning algorithms as given in Figure 1.0. The biggest score of $90 \%$ was achieved using the 1st method or process on feature extraction algorithm as shown in section 3rd mixed with Linear Support Vector Classifier on the analysis database. The similar correctness was taken by using Logistic regression for analyzing either positive reviews or negative reviews. The systems correctness lightly reduces by $5 \%$ when applying the Naive Bayes classifier.

\section{CONCLUSIONS AND FUTURE WORK}

In the research paper provides the many usages of several feature extraction techniques and classifiers for the classification of business text reviews by utilizing a wide dataset, The yelp challenge dataset which includes more than millions reviews . Our best classifiers are the Linear Support Vector Classifier Algorithm and SGD which obtained an efficiency of $94.4 \%$ using the first method proposed in the feature extraction algorithm. In terms of performance, their Logistic Regression and Naive Bayes classifiers give have lightly most unfavorable results.

Sentimental analysis means opinions, emotions, feelings, and expressions become a very challenging task in humans analysis. Sentiment analysis techniques proved to perform well for classifying sentiments of Yelp business reviews by taking into account the star rating which is given by the users. We thought that the accuracy of the system could still be improved the performance by implementing many usages of bigrams or trigrams, words, text, characters, or even part-of-speech features to distinguish between the same word features that are used for various part-of-speech. 


\section{REFERENCES}

1. B. Pang, L. Lee, and S. Vaithyanathan, "Thumbs up?: It Is Study Of sentiment classification using machine learning techniques," Proceedings of the ACL-02 conference on Empirical methods in natural language processing, vol. 10, pp. 79-86,2002

2. H. Minqing and B. Liu, "Opinion mining and summarizing customer reviews," in Proceedings of the tenth ACM SIGKDD international conference based on Knowledge discovery and data mining,2004

3. F. Mingming and M. Khademi, "Predicting a future business star in Yelp from its reviews text alone", arXiv preprint arXiv:1401.0864,2014

4. Po-Wei Liang, Bi-Ru Dai, "Opinion Mining on Social Media Sites", IEEE 14th International Conference on Mobile Data Management, Milan, Italy, June 3 - 6, 2013, pp 91-96, ISBN: 978-1- 494673-6068-5.

5. Pablo Gamallo, Marcos Garcia, "Citius: A Naive-Bayes alorgthim for Sentiment Analysis on English Tweets", 8th International Workshop on Semantic Evaluation (SemEval 2014), Dublin, Ireland, Aug 23-24 2014, pp171175.

6. Wan, X.."A Comparative Study of Cross-Lingual Sentiment Classification”. In Proceedings of the The 2012 IEEE/WIC/ACM International Joint Conferences on Web Intelligence and Intelligent Agent Technology-Volume 01 (pp. 24-31).IEEE ComputerSociety.2012.

7. D. Sanjiv, M. Chen, "Yahoo! for Amazon: Sentimental analyses extraction from small talk on the web", Management Science, pp. 1375-1388,2007.

8. D. Sanjiv, M. Chen, "Yahoo! for Amazon: Sentimental analysis extraction from small talk on the web", Management Science, pp. 1375-1388,2007.

9. A. Esuli and F. Sebastiani, "SentiWordNet:A Publicly Available Lexical Resource for Opinion Mining", Proceedings from International Conference on Language Resources and Evaluation (LR), 2006, pp.417-422

10. Elli, Maria Soledad, and Yi-Fan Wang. "Amazon Reviews, business analytics with sentimental analysis."2016

11. Elli, Maria Soledad, and Yi-Fan Wang. "Amazon Reviews, business analytics with sentiment analysis."2016

12. Xu, Yun, Xinhui $\mathrm{Wu}$, and Qinxia Wang. "Sentiment Analysis of Yelp,,s Ratings Based on Text or opinion mining Reviews."(2015).

13. Chen, Weikang, Chihhung Lin, and Yi-Shu Tai."Text-Based Rating Predictions on E-commerce sites (Amazon) Health \& Personal Care Product Review."(2015)

14. Text mining or opinion mining for yelp dataset task challenging; Mingshan Wang; University of California San Diego,(2017)

15. He, Ruining, and Julian McAuley. "Ups and downs: Modeling the visual evolution of fashion trends with oneclass collaborative filtering." Proceedings of the 25th International Conference on World Wide Web.International World Wide Web Conferences Steering Committee,2016.

16. L. Barbosa, J. Feng. "Robust Sentimental analysis Detection on Twitter from Biased and Noisy Data". COLING 2010: Poster Volume,pp.36-44.

17. Taboada, M., Brooke, J., Tofiloski, M., Voll, K., \&Stede, M.."Lexiconbasedmethods for sentiment analysis reviews". Computational linguistics, 2011:37(2),267-307. 